\title{
Poly(lactic-co-glycolic acid) Nanoparticles Loaded with Callistemon citrinus Phenolics Exhibited Anticancer Properties against Three Breast Cancer Cell Lines
}

\author{
Rashid Ahmed, ${ }^{1,2,3}$ Muhammad Tariq, ${ }^{1}$ Irfan S. Ahmad, ${ }^{2}$ Hanafy Fouly, ${ }^{2}$ Fakhar-i-Abbas, ${ }^{4}$ \\ Anwarul Hasan $\mathbb{D}^{3,5}$ and Mosbah Kushad ${ }^{6}{ }^{6}$ \\ ${ }^{1}$ Department of Biotechnology, Mirpur University of Science and Technology (MUST), Mirpur 10250, \\ Azad Jammu and Kashmir, Pakistan \\ ${ }^{2}$ Micro and Nanotechnology Laboratory, University of Illinois at Urbana Champaign, Urbana, IL, USA \\ ${ }^{3}$ Department of Mechanical and Chemical Engineering, College of Engineering, Qatar University, Doha 2713, Qatar \\ ${ }^{4}$ Bioresource Research Centre Islamabad, Islamabad, Pakistan \\ ${ }^{5}$ Biomedical Research Centre, Qatar University, Doha, Qatar \\ ${ }^{6}$ Department of Crop Sciences, University of Illinois at Urbana Champaign, Urbana, IL 61801, USA
}

Correspondence should be addressed to Anwarul Hasan; ahasan@qu.edu.qa and Mosbah Kushad; kushad@illinois.edu

Received 18 January 2019; Accepted 20 May 2019; Published 1 July 2019

Guest Editor: Amani Taamalli

Copyright (C) 2019 Rashid Ahmed et al. This is an open access article distributed under the Creative Commons Attribution License, which permits unrestricted use, distribution, and reproduction in any medium, provided the original work is properly cited. The publication of this article was funded by Qatar National Library.

\begin{abstract}
Fruit and vegetable diets rich in phenolic compounds reduce the risk of various cancers and offer multiple other health benefits due to their bioactivity and powerful antioxidant properties. However, the human health benefits of most phenolic compounds are restricted due to their limited aqueous solubility, low absorption, restricted passive cellular efflux, and poor gastrointestinal stability. Nanotechnology has been used to deliver various therapeutic drugs to specific targets overcoming many of the limitations of direct treatments. This study was designed to develop poly(lactic-co-glycolic acid) (PLGA) nanoencapsulated phenolic-rich extracts from Callistemon citrinus and berberine and to evaluate their effectiveness against extremely invasive MDA-MB 231, moderately invasive MCF-10A, and minimally invasive MCF-7 breast cancers. We have achieved about $80 \%$ encapsulation of phenolics from C. citrinus. Most encapsulated nanoparticles were polygonal with particles sizes of 200 to $250 \mathrm{~nm}$. Release of phenolics from encapsulation during storage was biphasic during the first week and then levelled off thereafter. Nanoencapsulated phenolics from C. citrinus extract, berberine, and combination of both enhanced their bioactivity against the three breast cancer cell lines by nearly 2 -fold. Growth inhibition of cells was a linear curve relative to phenolic concentration, with a maximum inhibition of nearly $100 \%$ at $0.1 \mathrm{mg} / \mathrm{ml}$ compared to control.
\end{abstract}

\section{Introduction}

Voluminous in vivo and in vitro studies have confirmed that diets containing fruits and vegetables, which are rich in phenolic phytochemicals, reduce the risk of several types of cancers and provide multiple human health benefits [1-3]. Recent research and review articles have demonstrated that dietary phenolic compounds reduce the risk of UV-induced oxidative and free radical damage, prevent cancers of the breast, stomach, prostate, and skin, and protect against inflammation, diabetes, and neurotoxicity $[2,4,5]$. It has been suggested that dietary phenolics deliver better preventive and therapeutic options, by improving phytonutrient bioavailability and enhancing drug activity, while exerting low toxicity, compared to conventional drug treatments [6].

The genus Callistemon consists of 34 species widely grown in several parts of the world. Leaves, inflorescence, 
and oils collected from this genus have for centuries been used in tribal medicine to treat gastrointestinal disorders, various pains, and infectious diseases [7]. Scientific evidence collected over the last few decades indicates that several Callistemon species contain bioactive compounds with medicinal properties against cardiovascular diseases and inflammation and anticancer and antidiabetic activities [7]. The medicinal properties of many of these species have been attributed to their rich content of polyphenolic antioxidants, flavonoids including flavanols, flavanones, terpenoids, and tannins, and several nonphenolic bioactive compounds including alkaloids, glycosides, and saponins $[7,8]$. Significant differences in polyphenolic concentrations and bioactivity were observed among different species within the Callistemon genus and between different plant parts of the same species with leaves of $C$. viminalis containing as high as $44 \%$ per dry weight polyphenols $[9,10]$.

Several studies have examined the antibacterial and antifungal properties and bioactivity of oil extracts from several Callistemon species including viminalis, comboynensis, lanceolatus, citrinus, rigidus, and linearis [11, 12]. For example, nitisinone extracted from C. rigidus was reported to lower tyrosinaemia type 1 , which can lead to buildup of tyrosine and its byproducts causing serious illnesses [7], while extracts from C. lanceolatus were reported to possess anticholinesterase activity [13]. However, limited research has been done on the anticancer properties of the nonoil bioactive compounds (primarily phenolics) found in Callistemon species. C. citrinus (Curtis) Skeels, commonly known as crimson or lemon bottlebrush, is very rich in phenolics and other bioactive compounds; however, there are very limited data on the potential of this species to reduce cancer $[14,15]$.

Significant advances have been reported in recent years in nanoparticle systems, especially polymeric nano/ microparticles used in cancer therapy [16]. Nanoparticles have been developed from various biocompatible and biodegradable materials that may be natural and/or synthetic and therefore display putative ability as carriers for treating various kinds of diseases, especially cancer [17]. In addition, polymeric nanoparticles are nontoxic and display a prolonged circulation potential and a wide payload spectrum for therapeutic agents. Synthetic polymers/copolymers such as PLGA, PLA, and PVA are preferred materials for the development of polymeric nanoparticles, as these systems can deliver drugs for days or even weeks compared to natural polymers, such as chitosan and sodium alginate nanoparticles, which have a shorter period of drug delivery and often require potentially toxic organic solvents [18]. Polymeric nanoparticles can be degraded enzymatically or nonenzymatically in vivo and thus produce biocompatible and safe byproducts that can be easily cleared from the body. Drugs encapsulated in polymeric nanoparticles are either dispersed inside the polymer matrix or attached/conjugated to polymer molecules allowing their release from nanoparticles upon their degradation [18].

Even though there is conclusive evidence to support the positive effects of phenolics on human heath, their low solubility and bioavailability may have limited their health benefits in many of the previous studies [19]. Nanoparticle delivery systems have successfully been used to encapsulate bioactive compounds and deliver them to intended targets in order to enhance their absorption and/or bioavailability $[20,21]$. The aim of this study was to utilize advances in nanotechnology to encapsulate phenolic-rich extracts from C. citrinus, examine stability and effectiveness of nanoencapsulated C. citrinus nanoparticles, and evaluate their effects on growth and proliferation of three types of breast cancer cell lines.

\section{Materials and Methods}

2.1. Materials. Biopolymer poly(lactic-co-glycolic acid) (PLGA) 50:50, berberine chloride hydrate (mol. wt. 371.9 Da), gallic acid, sodium carbonate, Folin-Ciocalteu reagent, 2,2-diphenyl-1-picrylhydrazyl (DPPH), and polyvinyl alcohol (PVA) (99.3-100\% hydrolyzed, average mol. wt. 85,000-124,000 Da) were purchased from Sigma-Aldrich (St. Louis, MO, USA). Acetone and methanol were purchased from Fisher Scientific Laboratory (NJ, USA), and potassium phosphate buffer was purchased from EMD Chemicals, Inc. (Gibbstown, NJ, USA). Human breast carcinoma (MCF-7, MCF-10A, and MD-MB 231) cell lines were purchased from American Type Culture Collection (Manassas, VA, USA). Dulbecco's modified Eagle medium, fetal bovine serum (FBS), and penicillin-streptomycin/ EDTA solution were purchased from Gibco (Carlsbad, CA, USA).

2.2. Tissue Sample Preparation. Bottlebrush Callistemon citrinus leaves and stems were collected from mature plants grown in Chakwal District of Punjab Province, Pakistan. Fresh tissue was frozen at $-70^{\circ} \mathrm{C}$, freeze-dried, and ground into fine powder. A subsample of $20 \mathrm{~g}$ freeze-dried tissue was mixed with $200 \mathrm{ml}$ methanol and homogenized, and the homogenate was placed on an orbital shaker (VWR, Hampton, NH, USA) for 12 hours. The mixture was filtered through a Whatman (\#4) filter paper (Marlborough, MA, USA), and the filtrate was concentrated in a rotary evaporator (Buchi Co., New Castle, DE, USA). Fresh extracts were prepared for each assay.

\subsection{Preparation and Loading of PLGA Nanoparticles.} Nanoparticles were prepared by a nanoprecipitation technique [22] with slight modifications as described by Pereira et al. [23]. An organic phase was formed when a fraction of $5.0 \mathrm{~mL}$ of the previously prepared methanolic tissue extracts was combined with $50 \mathrm{mg}$ PLGA prepared in acetone solution and stirred at $150 \mathrm{rpm}$ for 45 minutes. An aqueous phase of $1 \%$ polyvinyl alcohol (PVA) solution was prepared by dissolving $1.0 \mathrm{~g}$ of PVA in $100 \mathrm{ml}$ of ultrapure $\mathrm{H}_{2} \mathrm{O}$ and then heated and stirred to dissolve PVA. The organic fraction of the PLGA tissue extract was added to $20 \mathrm{~mL}$ PVA solution in a dropwise manner.

Similarly, a standard of $1.0 \mathrm{mg}$ of berberine chloride was dissolved in $1.0 \mathrm{ml}$ of methanol and $7 \mathrm{mg}$ of PLGA prepared in $1.0 \mathrm{ml}$ acetone. Berberine chloride solution was added to 
the PLGA solution and stirred for 45 minutes. The PLGAberberine mixture was added to another $20 \mathrm{~mL}$ PVA solution in a dropwise manner and stirred for 10 minutes at room temperature. Mixing of the organic phase with the aqueous phase while stirring resulted in aggregation of PLGA nanoparticles, which was prevented by adding $2.0 \mathrm{ml}$ purified $\mathrm{H}_{2} \mathrm{O}$. The solvents (acetone and methanol) were removed by rotary evaporation. Similarly, blank samples of PLGA nanoparticles were synthesized without the tissue extracts by adding only PLGA solution following the same steps outlined previously. The PLGA nanoparticles were centrifuged (Eppendorf centrifuge 5415C, Hamburg, Germany) at 14,000 rpm for 15 minutes and then collected and purified by ultrafiltration as previously described [24, 25]. PLGA nanoparticles were lyophilized by mixing synthesized nanoparticles with trehalose (EDM Chemicals, Philadelphia, PA, USA) at $1: 1$ ratio by pouring the PLGA nanoparticles into glass vials covered with a double layer of Parafilm. The PLGA nanoparticles were stored at $-80^{\circ} \mathrm{C}$ for 24 hours and then freeze-dried (Labconco, Kansas City, MO, USA).

\subsection{Scanning Electron Microscopy (SEM) of PLGA} Nanoparticles. The prepared nanoparticles were evaluated under a scanning electron microscope (Hitachi S-4800 FESEM, Japan) for their size, morphology, and surface properties. Nanoparticles were washed three times with distilled water, freeze-dried, and coated with gold palladium to improve electrical conductivity before imaging under SEM at $15 \mathrm{kV}$.

2.5. Nanoparticle Properties. PLGA nanoparticle diameter $(\varnothing$, size), surface property or zeta potential $(\zeta)$, and polydispersity index (PI), which determines nanoparticle penetration potential, aggregation capacity, and diffusion rate, were determined using Nano ZS90 Zetasizer (Malvern, UK) and Hitachi S-4800 scanning electron microscopy (SEM). PLGA nanoparticles were suspended in pure water in a cuvette (Malvern Panalytical, UK) and were sonicated to uniformly disperse the particles, in order to determine their size and any aggregate formation. Aggregation of PLGA nanoparticles was eliminated by filtering through a $0.2 \mu \mathrm{m}$ nylon filter (Acrodisc, Pall Corporation, Port Washington, NY, USA). The zeta potential of nanoparticles was determined by suspending PLGA nanoparticles in pure water and then transferring them into a zeta capillary cell (Malvern, Worcestershire, UK), which was loaded into a Malvern Zetasizer. The passage of the laser beam through PLGA nanoparticles and fluctuation in scattering intensity of nanoparticles produced the signal of an electrophoretic charge present on PLGA nanoparticles. All readings were recorded at $25^{\circ} \mathrm{C}$ and a scattering angle of $90^{\circ}$ after dilution of samples with pure water to prevent multiscattering. Polystyrene was used as a standard to measure light scattering at an angle of $173^{\circ}$. Each sample was measured in triplicate.

2.6. Total Phenolic Entrapment Efficiency within Nanoparticles. UV spectroscopy was used to determine the amount of polyphenol and polyphenol matrices of the C. citrinus extract, berberine, and C. citrinus extractberberine mixture entrapped into PLGA nanoformulations. The amount of PVA applied as a stabilizer and surfactant were kept constant at $1 \%$ during formulation of PLGA nanoparticles. Entrapment efficiency $(\eta \%)$ of total phenolics of the C. citrinus extract within nanoparticles was determined by measuring the UV absorbance of total phenolics entrapped within PLGA nanoparticles according to Pereira et al. [23] with a slight modification. The $C$. citrinus extract and berberine loaded PLGA nanoparticles were suspended in $95 \%$ methanol solution at a concentration of $1.0 \mathrm{mg} / \mathrm{ml}$ and placed in the dark for 30 minutes at $37^{\circ} \mathrm{C}$. The mixture was filtered through a $0.2 \mu \mathrm{m}$ nylon filter. A $100 \mu \mathrm{l}$ fraction of the filtrate was mixed with $20 \mu \mathrm{l}$ FolinCiocalteu reagent, $830 \mu \mathrm{l}$ purified water, and $50 \mu \mathrm{l}$ sodium carbonate solution. Samples were placed in the dark at room temperature for 30 minutes, and absorbance was recorded at $725 \mathrm{~nm}$ using a UV160U spectrophotometer (Shimadzu Corp., Kyoto, Japan). Gallic acid was used as a standard to estimate the total phenolic content in the encapsulated PLGA nanoparticles $[25,26]$ :

$$
\eta=\frac{\text { amount of total phenolics entrapped }}{\text { total phenolic quantity used for encapsulation }} \times 100 \text {. }
$$

2.7. In Vitro Release of Total Phenolics. The release of total phenolics from nanoparticles was estimated by combining lyophilized nanoparticles in a phosphate buffer saline $(\mathrm{pH}$ 7.2 ) at $1.0 \mathrm{mg} / \mathrm{ml}$, which was suitable to create a sink condition. The mixtures were incubated at $37^{\circ} \mathrm{C}$ in a water bath for 24 hours and then filtered through a $0.2 \mu \mathrm{m}$ Acrodisc nylon filter [25]. Later, the filtrate was collected, and total phenolics released were measured as described in Section 2.5.

2.8. Stability of PLGA Nanoparticles. The stability of PLGA nanoparticles was evaluated by measuring the amount of total phenolics released after one week of storage at $4^{\circ} \mathrm{C}$. In summary, $1.0 \mathrm{mg} / \mathrm{ml}$ subsamples of refrigerated PLGA nanoparticles were centrifuged at $3,000 \mathrm{rpm}$ for 3 minutes. The supernatants were collected, and the amount of total phenolics released was measured as previously described. The results were compared to total phenolics released by freshly prepared PLGA nanoparticles.

2.9. Anticancer Activity Determination. The anticancer activity of C. citrinus and berberine extract loaded PLGA nanoparticles was determined by a sulforhodamine B (SRB) colorimetric assay (Invitrogen, Carlsbad, California) according to a protocol outlined by Vichai and Kirtikara [27]. The protocol was used for cytotoxic screening of PLGA nanoparticles based on cell density measurement by estimation of protein content. Three cancer cell lines (MCF-7, MCF-10A, and MDA-MB 231) were used to study the effect of PLGA nanoparticles and unencapsulated form of 
C. citrinus and berberine. The human hormone-dependent breast cancer cell lines MCF-7 and MCF-10A were cultured in Dulbecco's modified Eagle medium (Gibco, Carlsbad, CA, USA), and MDA-MB 231 hormone-independent breast cancer cell lines were cultured in Leibovitz's L-15 medium (Sigma-Aldrich) in T75 cell culture flasks (Thermo Fisher Scientific), supplemented with $10 \%$ fetal bovine serum and $1 \%$ penicillin-streptomycin/EDTA solution (Gibco, Carlsbad, CA, USA). Cultured cells were immediately transferred to a 96-well plate (Thermo Fisher Scientific) and incubated overnight to a rate of about $1 \times 10^{4}$ cell density per well, and the percentage of growth inhibition of breast cancer cell lines was measured after a period of 48 hours.

Tested PLGA nanoparticles loaded with $C$. citrinus and berberine and free extracts from the same treatments ranged from 0.0008 to $0.1 \mathrm{mg} / \mathrm{ml}$. Samples were placed in 96-well plates, incubated under 5\% $\mathrm{CO}_{2}$-enriched air and $100 \%$ relative humidity at $37^{\circ} \mathrm{C}$ for 48 hours, fixed by adding $50 \mu \mathrm{l}$ of cold $10 \%(\mathrm{w} / \mathrm{v}) \mathrm{TCA}$, and then stored at $4^{\circ} \mathrm{C}$ for 1 hour. Each sample well was washed four times with distilled water and then dried by a purified air stream at room temperature.

The staining step was performed by adding $100 \mu \mathrm{L}$ of $0.57 \%(\mathrm{w} / \mathrm{v})$ sulforhodamine B (SRB) dye to each sample well. Treated samples were incubated at room temperature for 30 minutes and then washed four times with $100 \mu \mathrm{l}$ of $1 \%$ $(\mathrm{v} / \mathrm{v})$ acetic acid to remove the unbound dye. Finally, $100 \mu \mathrm{l}$ of $10 \mathrm{mM}$ Tris base ( $\mathrm{pH}$ 10.5) was added to each sample well, and the plates were placed on a shaker for 3 minutes. The absorbance of each sample was measured using a spectrophotometer set at $490-630 \mathrm{~nm}$, and the growth inhibition percentage was determined using the following formula:

$$
\text { growth inhibition } \begin{aligned}
(\%)= & {\left[\frac{\text { mean } \mathrm{OD} \text { of sample }- \text { mean } \mathrm{OD}_{\mathrm{b}}}{\text { mean OD of control }- \text { mean } \mathrm{OD}_{\mathrm{b}}}\right] } \\
& \times 100,
\end{aligned}
$$

where $\mathrm{OD}_{\mathrm{b}}$ is the OD for a blank sample.

2.10. Statistical Analysis. Results are expressed as mean \pm standard deviation (SD). Data were analyzed by one-way analysis of variance, and differences among the means of groups were analyzed by an unpaired, two-sided Student's $t$ test. Differences were significant at $P<0.05$.

\section{Results and Discussion}

\subsection{Preparation and Characterization of PLGA} Nanoparticles. In our study, PLGA nanoparticles were synthesized by a nanoprecipitation technique, as outlined in Figure 1. Polyvinyl alcohol (PVA), a biodegradable and biocompatible synthetic biopolymer, was used as a stabilizer for PLGA nanoparticles. By using PVA as a stabilizer and PLGA as a carrier, we were able to obtain high yield of nanoparticles. PLGA has been widely used for drug delivery applications of hydrophobic drugs such as polyphenols. It is also biodegradable, biocompatible, and a highly stable polymer. It has also been approved by the USA Food and Drug Administration.

3.2. Scanning Electron Microscopy. Sizes of PLGA nanoparticles loaded by berberine, $C$. citrinus extract, and mixtures of berberine and $C$. citrinus extract were $250.7 \pm 0.06$, $278.8 \pm 0.007$, and $274.8 \pm 0.028 \mathrm{~nm}$, respectively (Table 1 ). Nanoparticle shapes observed under SEM were polygonal (hexagonal) with diverse sizes, with unique morphological features (Figures 2(a) and 2(b)), as compared to a previously reported formulation [24]. Various studies have demonstrated that tailoring of the shape of nanoparticles could improve their effectiveness. For example, it was reported that polygonal nanoparticles have more antibacterial activity against $E$. coli than triangular and spherical nanoparticles [28]. In another study, it was observed that oblong-shaped nanoparticles improved targeted delivery of antibody nanoformulations [29]. Nanorods coated with trastuzumab exhibited a $66 \%$ increase in binding and uptake potential by BT-474 breast cancer cell lines compared to an equal dose of spherical nanoparticles, resulting in a 5-fold growth inhibition potential against BT-474 cancer cells [30]. While oblate nanoparticles exhibited a better degree of adherence to mammalian cells and a higher drug-carrying capacity compared to spherical particles [31], polygonal nanoparticles were reported in an earlier study to exhibit a prolonged stay in blood stream due to uptake failure of macrophages [32]. This mechanism may lead to enhanced growth inhibition due to higher penetration through cell membranes, subsequently releasing polyphenols into the cytoplasm, which contains various organelles that could be affected by the nanoformulations [25].

The enhanced growth inhibition feature of polygonal nanoparticles was observed during their cytotoxic effect on various types of cancer cell lines as these nanoformulations penetrate more efficiently into cells due to their morphological matrix $[33,34]$. In addition to their morphology, nanoparticles' surface charges also determine their potency. The effectiveness of positively charged PLGA nanoparticles was higher than that of PLGA nanoparticles bearing negative charges [35]. Nanoparticles' surface charges also affect their aggregation. Aggregation of nanoparticles developed in this study was significantly reduced by filtration followed by addition of an excess solvent. Our PLGA nanoparticles were more efficiently separated when they had higher surface charges and tended to aggregate when they had lower surface charges.

3.3. Characterization of Nanoparticles. Nanoparticle size and polydispersity index (PDI) define efficient delivery of loaded materials. Both nanoparticle size and PDI determine drug loading and drug release profiles, stability, toxicity, and targeting potential, which are main requirements for an ideal cargo carrier system. The nanoparticle size obtained in the current study ranged from 200 to $250 \mathrm{~nm}$ (Table 1), which is considered an optimum size range for PLGA nanoparticles [36]. Zeta potential is another important characteristic that determines cytotoxicity 


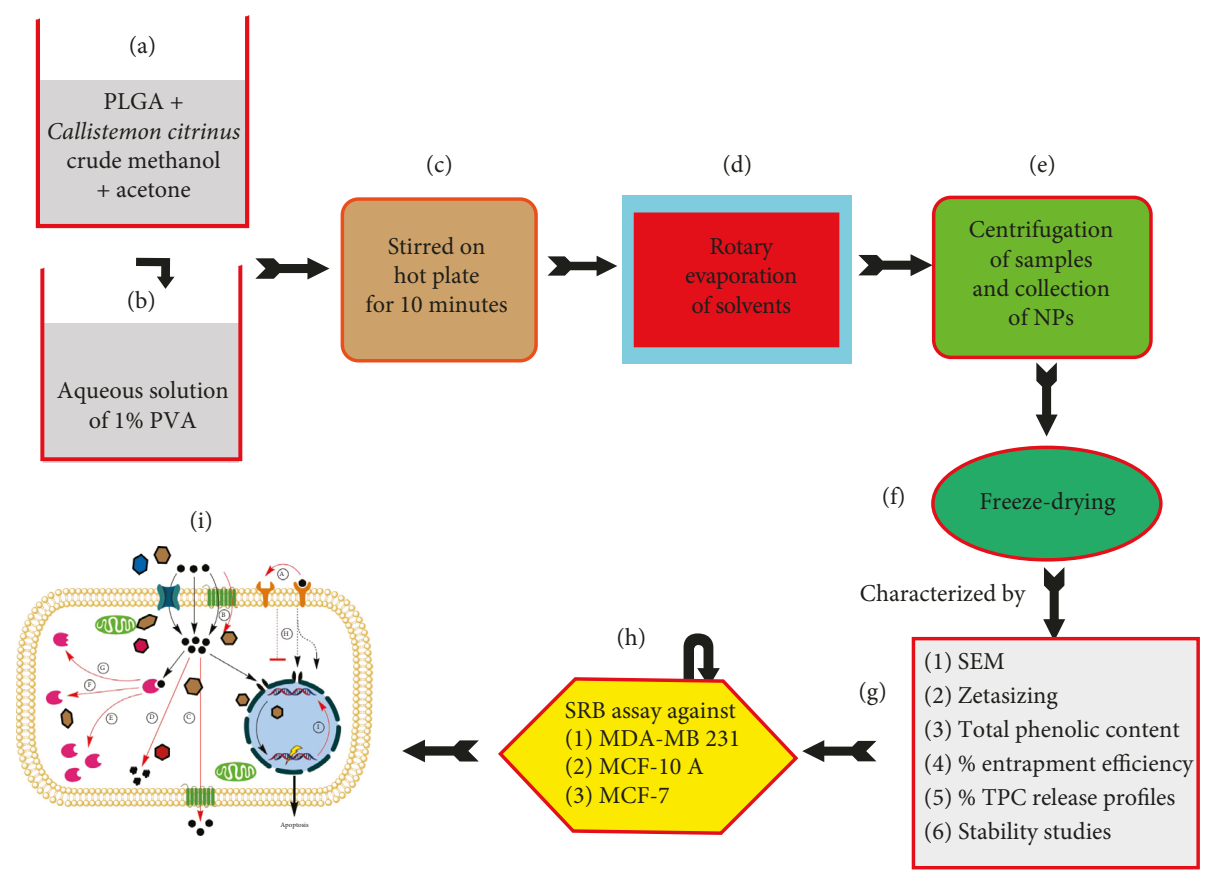

Figure 1: Flow chart for the synthesis and characterization of C. citrinus extract-loaded PLGA nanoparticles. (a) Mixing of C. citrinus extract solution with PLGA solution. (b) Preparation of 1\% PVA solution. (c) PLGA solution containing C. citrinus extract was constantly stirred at $150 \mathrm{rpm}$ for 30 minutes, leading to precipitation of PLGA NPs. (d) The solvent was evaporated by rotary evaporation. (e) Collection of PLGA nanoparticles by centrifugation at $14,000 \mathrm{rpm}$. (f) Collection of PLGA NPs was freeze-dried and stored at $-4^{\circ} \mathrm{C}$. (g) Characterization of PLGA-loaded nanoparticles by scanning electron microscopy, zetasizing, total content in extracts, loading of total phenolics (TP) into PLGA nanoparticles, and percentage TP release studies and stability profiles. (h) Assessment of growth inhibitory effect of PLGA NPs and their free counterparts against MDA-MB 231, MCF-10A, and MCF-7 breast cancer cells. (i) Mode of action of PLGA NPs on model cells.

TABLE 1: Estimates of zeta diameter, polydispersity index, and zeta potential of nanoformulations of BBR PLGA, CCE PLGA, and CCE BBR PLGA.

\begin{tabular}{lccccc}
\hline NPs & Incubation temperature $\left({ }^{\circ} \mathrm{C}\right)$ & Zeta diameter $(\mathrm{nm})$ & Aggregation $(\%)$ & Polydispersity index & Zeta potential $(\mathrm{mV})$ \\
\hline BBR PLGA & 25 & $250.6 \pm 0.08$ & 0 & $0.361 \pm 0.06$ & $-8.50 \pm 0.06$ \\
CCE PLGA & 25 & $278.8 \pm 0.00$ & 0 & $0.545 \pm 0.07$ & $-20.06 \pm 0.10$ \\
CCE BBR PLGA & 25 & $274.8 \pm 0.02$ & 0 & $0.256 \pm 0.04$ & $-5.80 \pm 0.09$ \\
\hline
\end{tabular}

Abbreviations: BBR, berberine; CCE, Callistemon citrinus extract; PLGA, poly(lactic-co-glycolic acid); NPs, nanoparticles. Data points represent mean \pm SD $(n=3)$.

and clumping of loaded PLGA nanoparticles. The surface charge of PLGA nanoparticles loaded with berberine and a combination of berberine and C. citrinus was $-8.50 \pm 0.06$ and $-5.80 \pm 0.09$, respectively, whereas the surface charge of PLGA nanoparticles loaded with C. citrinus alone was 2.4- to 3.5-fold lower than that loaded with berberine and mixture of berberine plus C. citrinus. Since nanoparticles' surface charge plays a significant role in inhibition of cancer growth [35], PLGA nanoparticles loaded with berberine plus $C$. citrinus could be more cytotoxic or more effective against cancer cells compared to PLGA nanoparticles loaded with C. citrinus alone. The PDI data in Table 1 and size distribution in Figures 2(c) and 2(d) show that the PLGA nanoparticles were of diverse sizes. Formation of diverse-sized PLGA nanoparticles is advantageous due to variation in their diffusion rates with smaller nanoparticles having higher diffusion than larger nanoparticles.
3.4. Total Phenolics and Their Entrapment in LPGA Nanoparticles. Berberine, an alkaloid antioxidant, reacted positively and linearly with the phenolic assay, but at a rate lower than that of $C$. citrinus phenolics. The total phenolic content of berberine, C. citrinus, and the mixture of $C$. citrinus and berberine was $1942.46 \pm 0.01,609.35 \pm 0.02$, and $1979.58 \pm 0.006 \mu \mathrm{g} / \mathrm{ml}$, respectively (Figure $3(\mathrm{a})$ ). The data obtained suggest that phenolic concentration in the C. citrinus extract and C. citrinus extract plus berberine was 4 -fold higher than that in berberine alone. Numerous articles have linked antioxidant properties of total phenolics to human health benefits but have very little information on the health benefits of their entrapment in nanoformulations.

Total phenolic entrapment of about $88 \%$ was observed in PLGA nanoparticles loaded with a blend of C. citrinus and berberine, followed by $82 \%$ entrapment from C. citrinus extract alone and only about $23 \%$ entrapment from berberine alone (Figure 3(b)). The solubility of the entrapped 


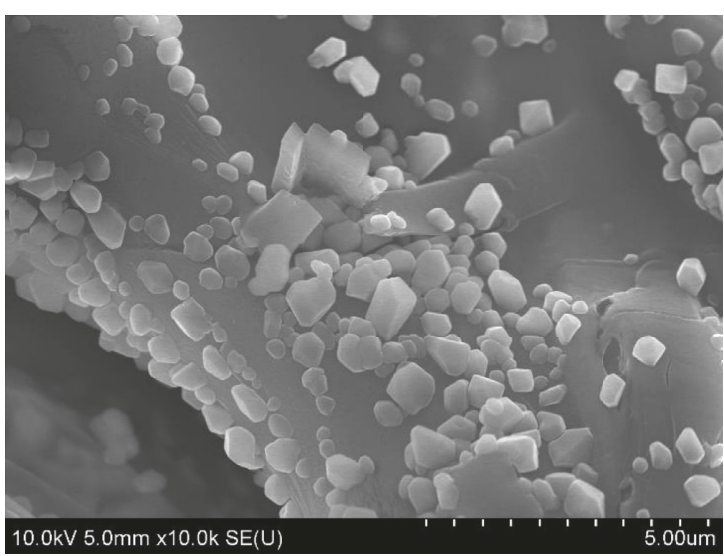

(a)

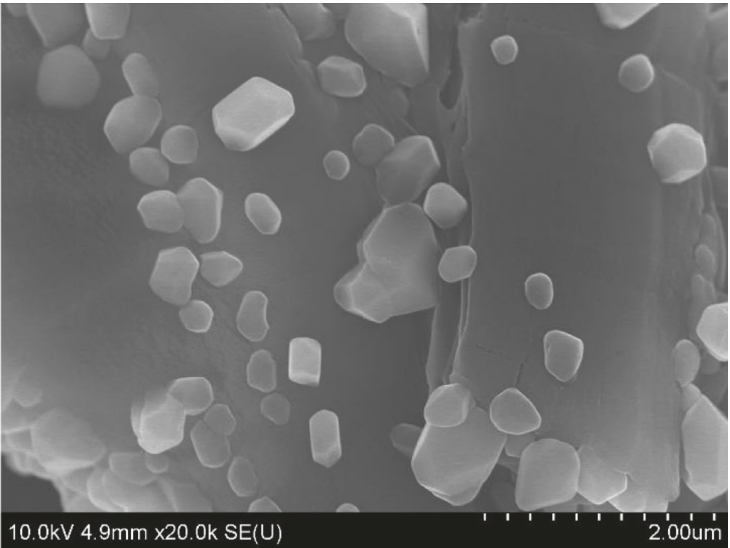

(c)

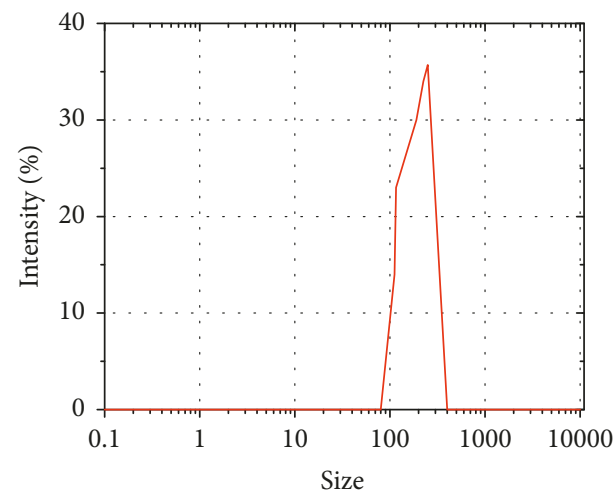

— Intensity

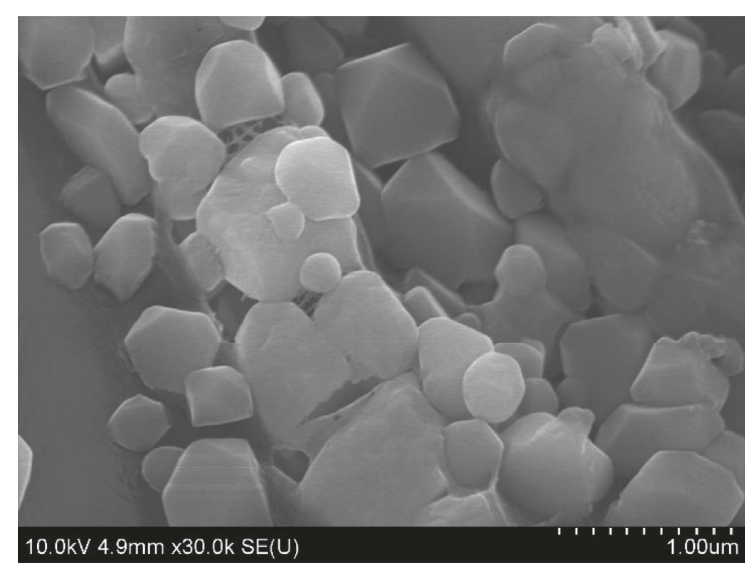

(b)

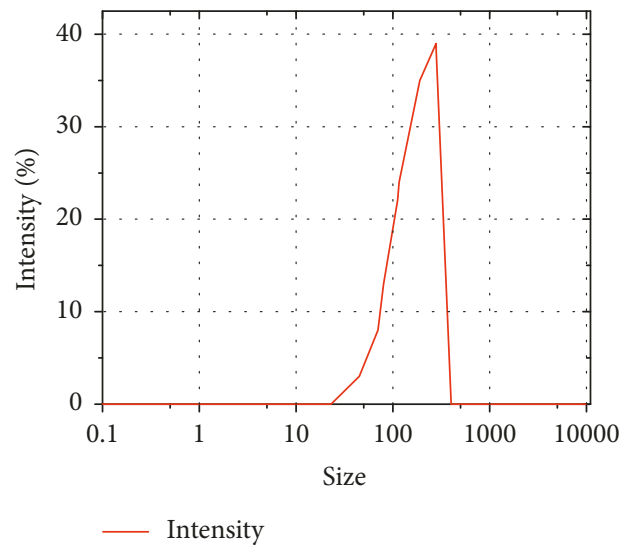

(d)

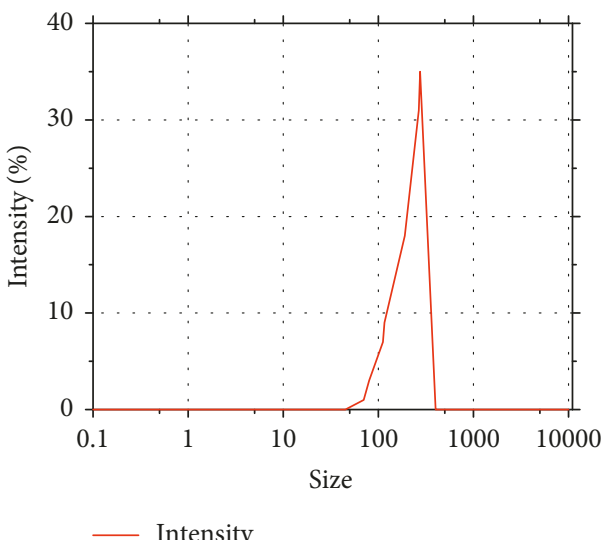

(f)

Figure 2: Size distribution images of PLGA NPs obtained through Malvern Zetasizer and Hitachi S-4800 scanning electron microscopy synthesized by a nanoprecipitation technique. SEM images of (a) C. citrinus, (b) berberine, and (c) C. citrinus + berberine nanoparticles. Histograms of (d) C. citrinus, (e) berberine, and (f) C. citrinus + berberine nanoparticles.

material in water determines the percentage of entrapment into PLGA nanoparticles as extracts having more solubility will move out into the aqueous phase from the organic phase during synthesis of PLGA nanoparticles, resulting in a decrease in percentage entrapment efficiency as described by Pereira et al. [37]. The entrapment of berberine and C. citrinus extracts into PLGA is consistent with the encapsulation of the hydrophobic extract of guabiroba fruit which was reported at between about 84 and 99\% encapsulation into PLGA nanoparticles [38].

3.5. Percent Release of Total Phenolics from Loaded Nanoparticles. Release of total phenolics from PLGA nanoparticles was carried out at $\mathrm{pH} 7.4$ and $37^{\circ} \mathrm{C}$, resembling conditions of the gastric juices in the human body [39]. Data 


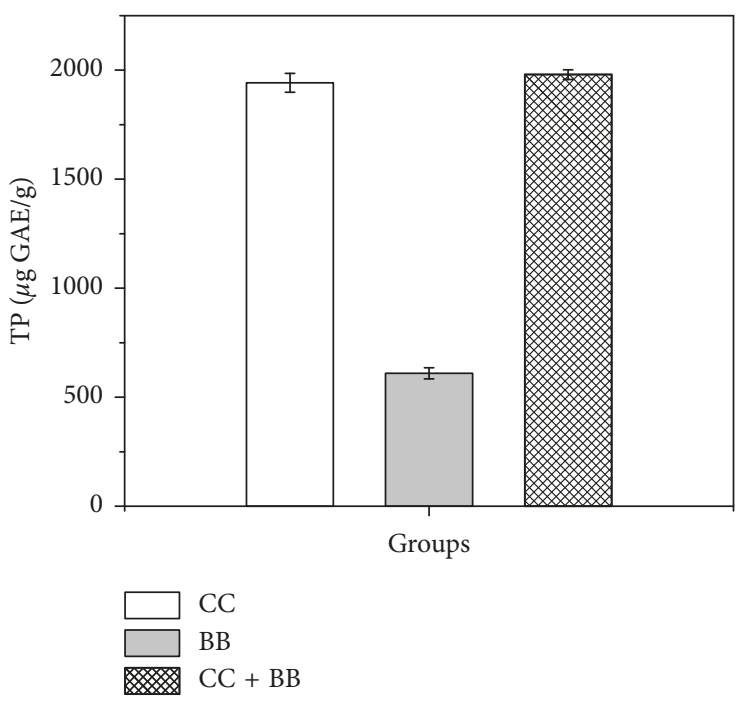

(a)

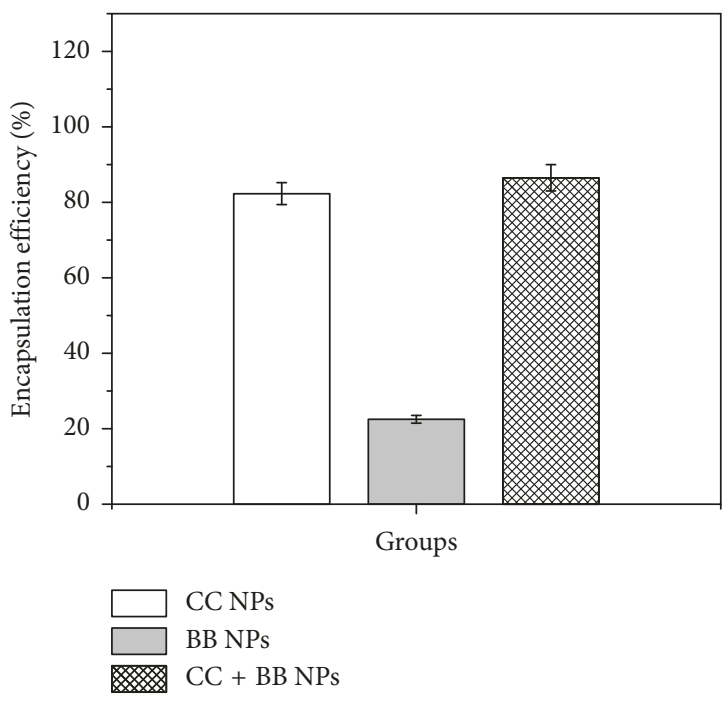

(b)

Figure 3: (a) Total phenolic content of C. citrinus (CC), berberine (BB), and C. citrinus extract + berberine (CC + BB) encapsulated in PLGA nanoparticles. (b) Percent encapsulation efficiency $(\eta \%)$ of PLGA nanoparticles loaded with $C$. citrinus, berberine, and C. citrinus + berberine. Data are represented as mean $\pm \mathrm{SD}(n=3)$.

in Figure 4(a) show linear curve responses with fast releases of berberine, $C$. citrinus extract, and mixture of $C$. citrinus and berberine from PLGA nanoparticles within the first $24 \mathrm{~h}$, reaching their peaks at 7 days and then levelling off to negligible levels thereafter. The immediate fast release of berberine and $C$. citrinus extract could be attributed to rapid desorption and subsequent release from the outer boundary of PLGA nanoparticles as previously described during chlorambucil encapsulation [40]. All three treatments exhibited a similar pattern of release with encapsulated berberine being significantly higher than the other two treatments during the first 7 days, while combination of berberine and C. citrinus release was significantly lower than the other two treatments during the remainder of the study period (Figure 4(a)). The slow and sustained release of total phenolics was due to difference in their concentration within PLGA nanoparticles and to the phosphate saline buffer. The change in sink conditions significantly affected the release profile of total phenolics in vivo where it was enhanced due to rapid degradation of PLGA nanoparticles [39].

3.6. Stability of PLGA Nanoformulations during Storage. Total phenolic release profiles in Figure 4(b) show that the prepared nanoformulations were very stable. Leakages from prepared PLGA nanoparticles containing berberine, mixture of berberine and $C$. citrinus phenolics, and $C$. citrinus phenolics alone showed less than $2 \%$ reduction over a 28 -day storage period at $4^{\circ} \mathrm{C}$ (Figure $4(\mathrm{~b})$ ). The stability of these nanoformulations may be due to the presence of a PVA coating barrier, allowing for better formulation retention. The low percent leakage of phenolics over the 28-day storage suggests that the prepared PLGA nanoformulations are a very stable carrier making them suitable for drug delivery applications. The stability of the encapsulated liposomal formulations reported in this study was very similar to that observed using curcumin under similar storage conditions [41], which indicates that storage of PLGA nanoparticles has no effect on their retention of polyphenols, and thus, they can be used as stable carrier systems for berberine and $C$. citrinus extract.

\subsection{Cytotoxic Activity of Nanoformulations against Breast} Cancer Cell Lines. The nanoformulation technique presented in this study is a useful tool for loading of complex extracts into polymeric nanoparticles to evaluate their anticancer potential for in vitro trials. Recent studies have used nanoencapsulation of a single or multiple bioactive compounds to evaluate their anticancer effectiveness against several types of cancers in vitro [42, 43]. Sampath et al. [44] examined the bioactivity of $C$. citrinus extracts against skin carcinoma A431 and human keratinocyte HaCaT cell lines. They attributed the apoptotic effect of $C$. citrinus extracts to their content of the monoterpenoid 1,8-cineole, which they reported to enhance the expression of p53, a tumorsuppressing protein. Similarly, essential oils extracted from C. citrinus leaves and flowers exhibited highly significant growth inhibition of A549 cells and C-6 cancer cells ( $61 \%$ and $69 \%$, respectively) but had no effect on growth of normal cells.

Data in Figures 5(a)-5(d) of nanoformulated and nonformulated treatments at different concentrations show different growth inhibition efficacies against the tested breast cancer cell lines. Figure 5(b) shows that, at $0.1 \mathrm{mg} / \mathrm{ml}$, a mixture of nanoformulated berberine and $C$. citrinus induced the most potent inhibition (33\%) of MDA-MB 231 cells. In contrast, the same concentration in nonformulated form induced only about $12 \%$ growth inhibitory, suggesting that encapsulation resulted in nearly a 3 -fold increase in effectiveness of the 


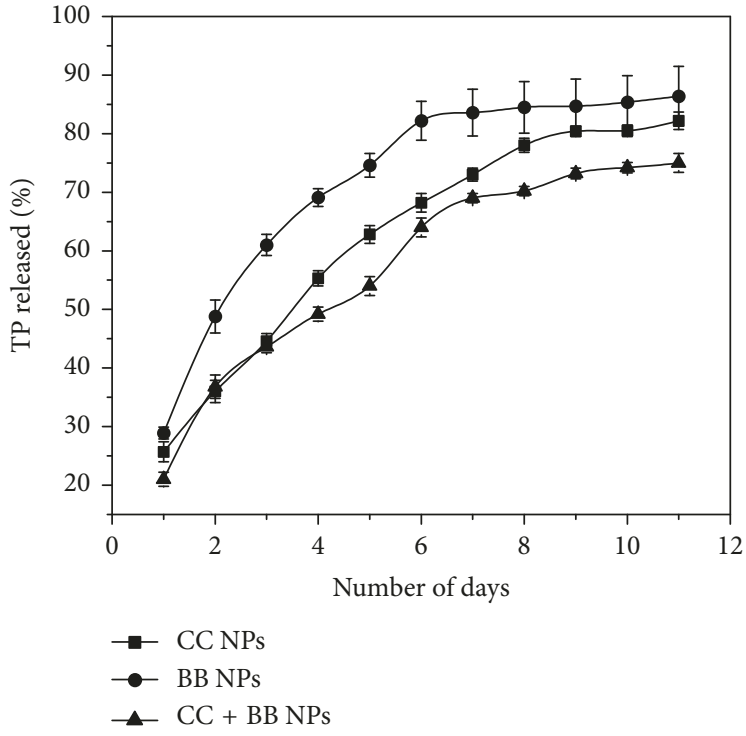

(a)

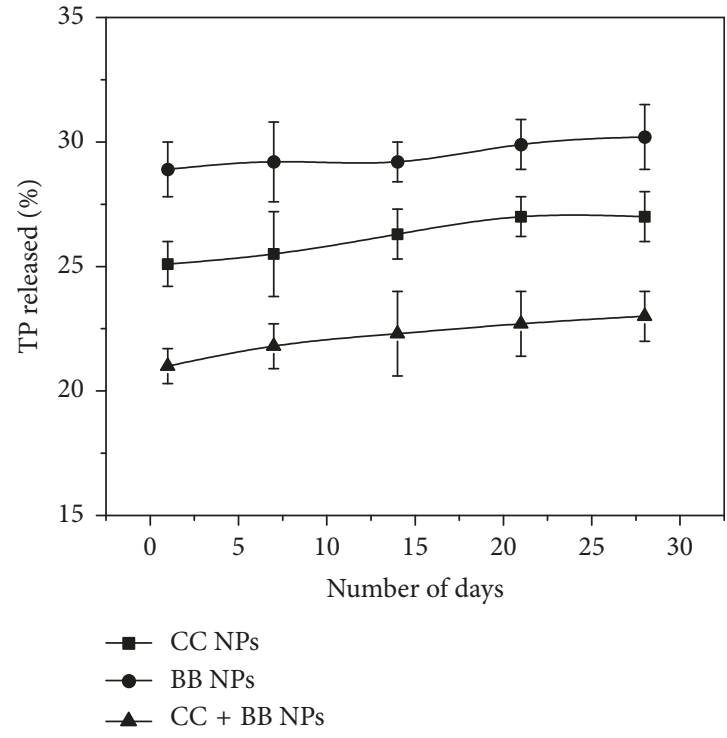

(b)

Figure 4: (a) Percentage release of TP of CC-, BB-, and CC + BB-loaded PLGA NPs over a period of 11 days under conditions resembling the human gastrointestinal tracts. (b) Percent release of TP from BB PLGA NPs (closed circle), CC PLGA NPs (closed square), and CC + BB PLGA NPs (closed triangle) over a period of 28 days in cold storage at $4^{\circ} \mathrm{C}$. Data represent mean $\pm \mathrm{SD}(n=3)$.

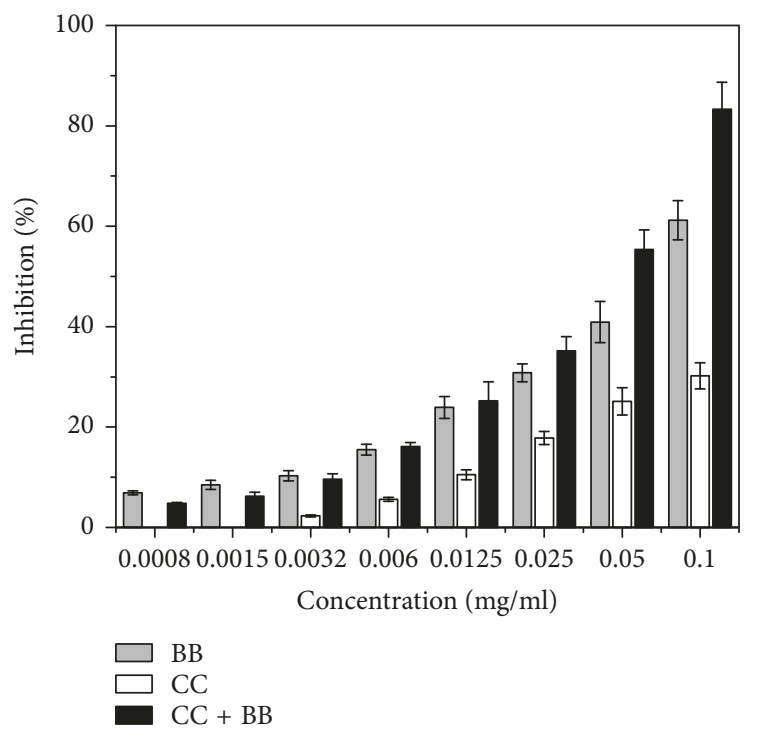

(a)

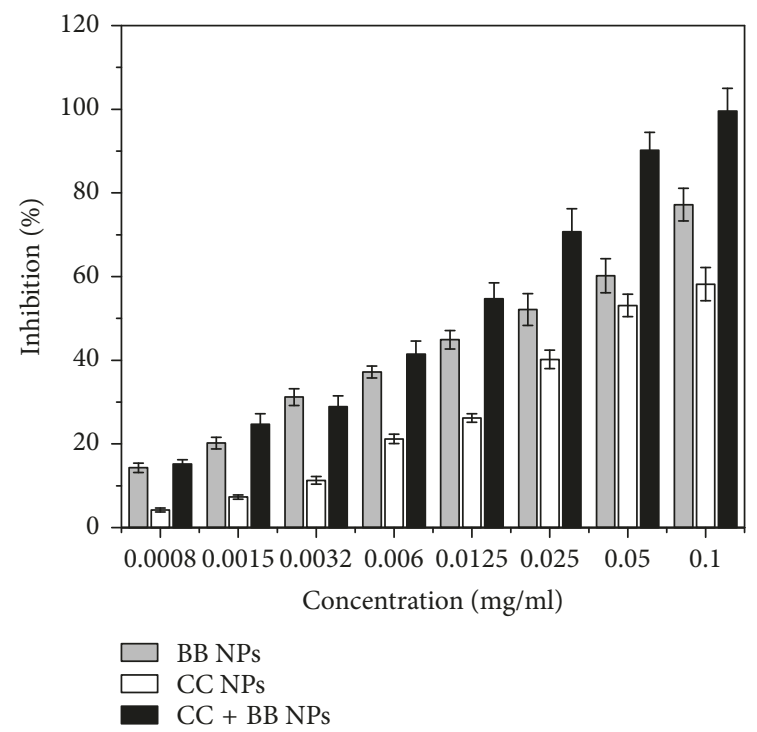

(b)

FIGURE 5: Continued. 


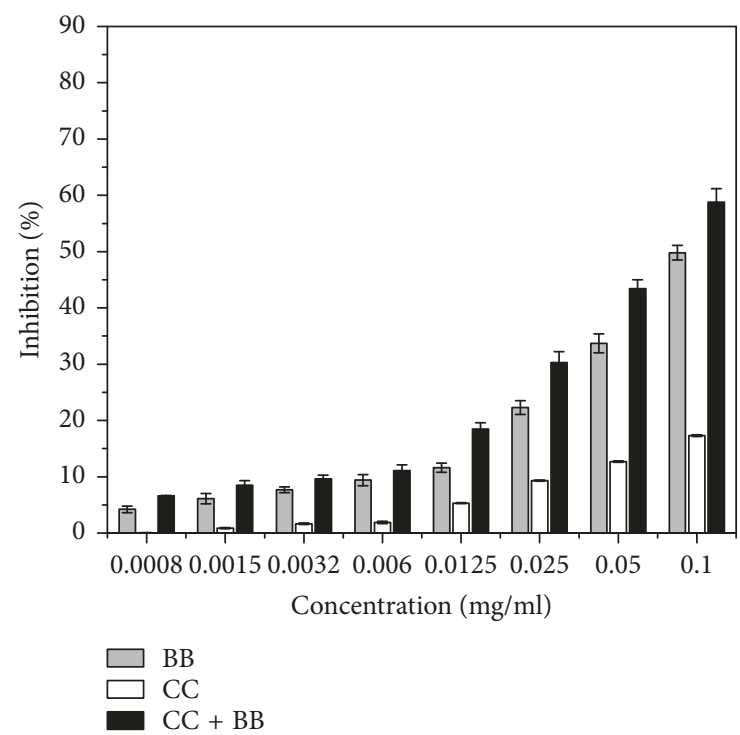

(c)

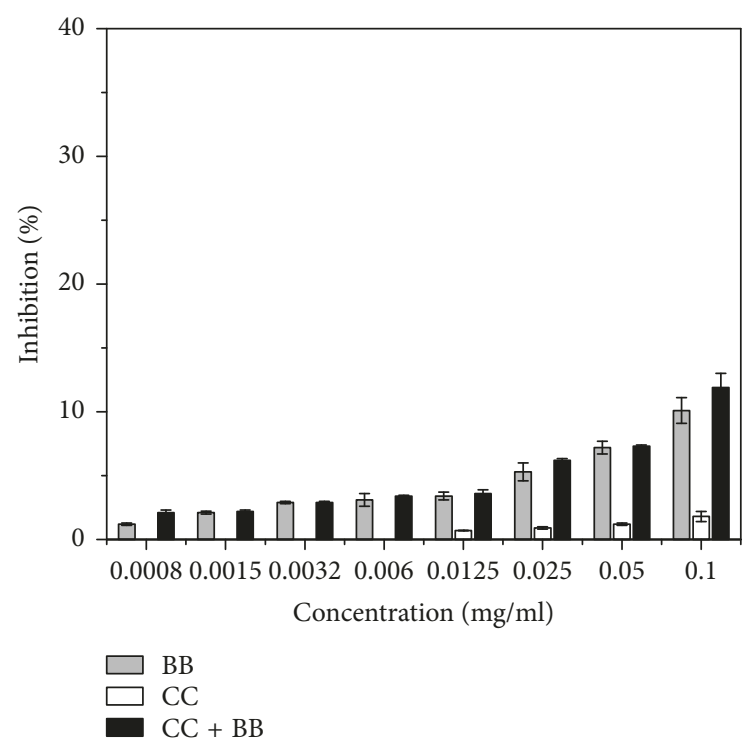

(e)

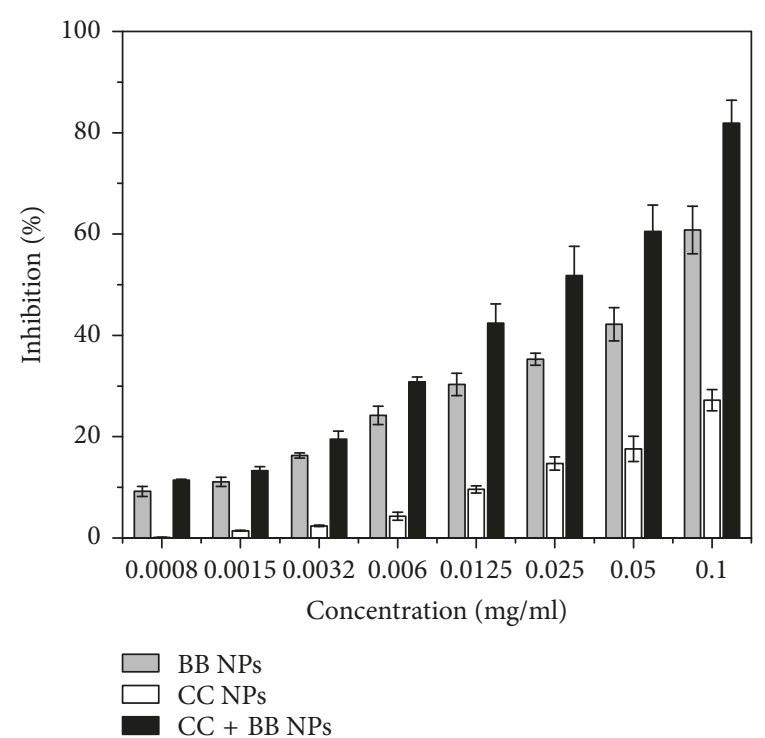

(d)

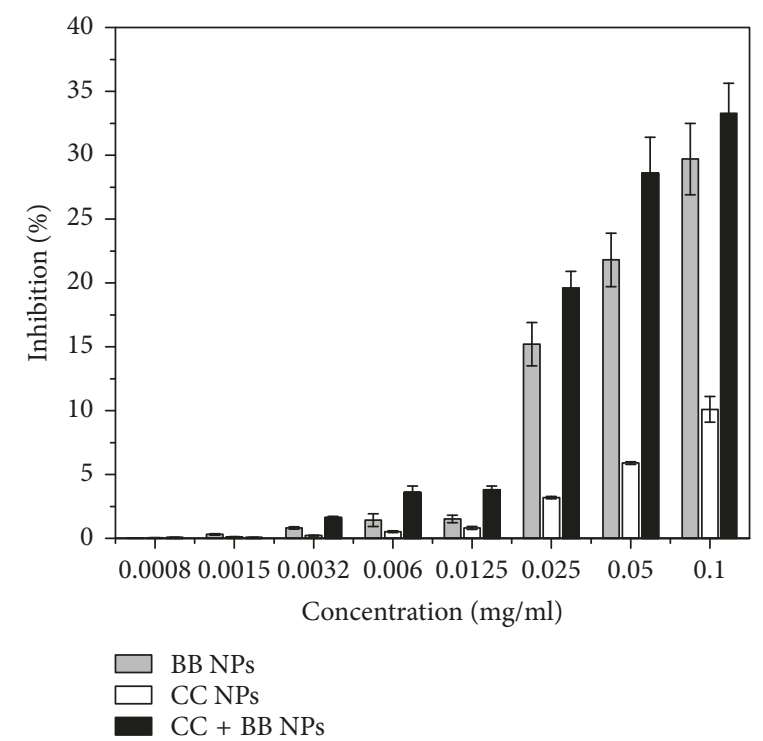

(f)

Figure 5: (a) Cytotoxic effects of BB, CC, and CC + BB extracts against MDA-MB 231 breast cancer cell lines. (b) Cytotoxic activity of BB PLGA NPs, CC PLGA NPs, and CC + BB PLGA NPs against MDA-MB 231 breast cancer cell lines. (c) Cytotoxic effects of BB, CC, and $\mathrm{CC}+\mathrm{BB}$ extracts against MCF-10A breast cancer cells. (d) Extract-loaded PLGA nanoparticles against MCF-10A breast cancer cells. (e) Cytotoxic effects of BB, CC, and CC + BB extracts against MCF-7 cancer cells. (f) Cytotoxic effects of BB-, CC-, and CC + BB loaded PLGA NPs against MCF-7 breast cancer cells (mean \pm SD, $n=3$ ).

nanoformulated treatments (Figure 5(b)). The cytotoxicity of free and encapsulated $C$. citrinus forms reported in this study is in agreement with that reported in other studies [45], showing a marked difference in their anticancer potential. Contrary to an earlier study [46], we have observed a marked increase in effectiveness of the treatments against MDA-MB 231 breast cancer when their concentrations were increased (Figures 5(a) and 5(b)). The effect of the treatments against MCF-10A, a more invasive cancer cell line, is shown in Figures 5(c) and 5(d). The patterns of MCF-10A response to the treatments' concentrations were similar to those for MDA-MB 231; however, at $0.1 \mathrm{mg} / \mathrm{ml}$ nonformulated and nanoformulated doses, the rate of inhibitions was about 2.5- and 5-fold higher, respectively, than those for $\mathrm{MD}-\mathrm{MB} 231$. The highest anticancer activities of the treatments were observed against MCF7 cell lines (Figures 5(e) and 5(f)). More than 83\% and nearly $100 \%$ cell growth inhibitions were observed in the nonformulated and nanoformulated treatments at $0.1 \mathrm{mg} / \mathrm{ml}$, respectively (Figures 5(d) and 5(f)).

Treatment of cancer cells with $C$. citrinus extracts induced higher growth inhibition compared to berberine in both nonformulated and nanoformulated forms. However, cancer cell growth inhibition was significantly higher when a mixture of both materials was used in either nanoformulated 


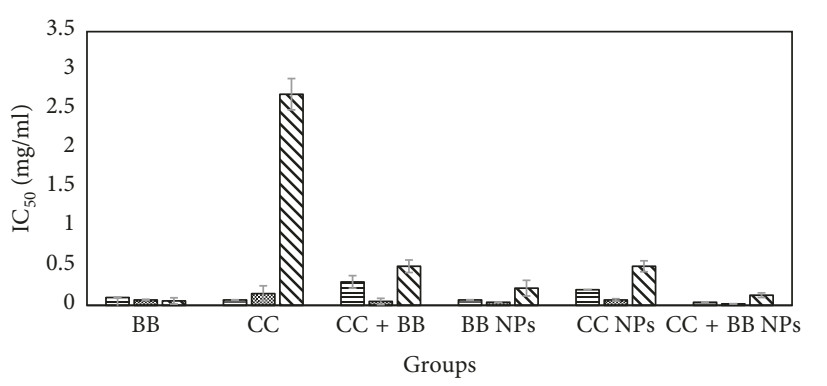

目 MCF-10A

MCF-7

ब MDA-MB 231

Figure 6: Values of IC $_{50}$ of MDA-MB 231, MCF-7, and MCF-10A breast cancer cell lines treated with nanoformulated and nonformulated $\mathrm{BB}, \mathrm{CC}$, and $\mathrm{CC}+\mathrm{BB}$ extracts (mean $\pm \mathrm{SD}, n=3$ ).

or nonformulated forms. This combination was highly effective against MCF-7, intermediately effective against MCF$10 \mathrm{~A}$, and slightly effective against MDA-MB 231 cancer cell lines. Synergistic effects were observed between the tea polyphenols theaflavin-3-3'-digallate, ascorbic acid, and (-)-epigallocatechin-3-gallate [47]. In contrast, PLGA nanoparticles loaded with berberine alone were minimally effective. Results of this study support the previous study using MCF-7 breast cancer cell lines [24]. Thus, PLGA nanoformulations loaded with complex polyphenol matrices could provide effective treatment to reduce and/or inhibit the proliferation of less invasive breast cancer cells. These results highlight two important observations: (a) significant inhibition of cancer cell growth when extracts were encapsulated into PLGA nanoparticles and (b) higher breast cancer cell inhibition when cells were treated with a combination of berberine plus C. citrinus.

Another important observation was the correlation between the degree of invasiveness of a cancer cell line and the degree of its growth inhibition by the treatment. For example, C. citrinus and berberine PLGA loaded nanoparticle treatment had minimal effects against the growth of the extremely metastatic MDA-MB 231 cancer cell line (Figure 6), while very effective against the other two less invasive lines. Our data confirm earlier observations by Kim et al. [48] using berberine against the same cell lines. In another study [49], treatment of MDA-MB 231 cancer cell lines with magnolol, a natural compound, significantly inhibited cell growth by downregulating the matrix of metalloproteinase-9 (MMP-9), an enzyme required for invasion and tumor metastasis. In our study, nanoformulated C. citrinus extract plus berberine showed a more pronounced growth inhibition of MCF-7 cells, which suggests that the degree of invasiveness/proliferation is related to growth inhibition. A similar pattern of growth inhibition was demonstrated against MDA-MB 231 and T47D cells using metformin in nanoencapsulated and free forms [50].

\section{Conclusion}

Nanoencapsulation of C. citrinus into PLGA nanoparticles enhanced its bioactivity against MDA-MB 231, MCF-10A, and MCF-7 breast cancer cell lines by about 2 -fold. In addition, the combination of $C$. citrinus extracts with berberine further increased their cytotoxic potential in free and encapsulated forms. Data suggest that nanoencapsulation of anticancer compounds either alone or in combination with other bioactive compounds is a valuable tool for cancer chemotherapy.

\section{Data Availability}

Data used to support the findings of this study are included in the article.

\section{Conflicts of Interest}

The authors declare that they have no conflicts of interest. Additionally, University of Illinois is an Affirmative Action/ Equal Opportunity Employer dedicated to building a community of excellence, equity, and diversity.

\section{Acknowledgments}

Support for this research was provided to RA by the Higher Education Commission (HEC) of Pakistan under International Research Support Initiative Program (IRSIP), M. M. Kushad by the Hatch Fund, and A. Hasan by the Qatar National Research Fund (grant no. NPRP10-0120-170211). The publication of this article was funded by Qatar National Library. Technical and training support was provided by Dr. Angana Senpan and staff at the Bionanotechnology, Micro and Nanotechnology, Center for Nanoscale Science and Technology, and the Frederick Seitz Materials Research Laboratories at University of Illinois at Urbana/Champaign, IL, USA.

\section{References}

[1] C. Lee-Chang, M. Bodogai, A. Martin-Montalvo et al., "Inhibition of breast cancer metastasis by resveratrol-mediated inactivation of tumor-evoked regulatory B cells," Journal of Immunology, vol. 191, no. 8, pp. 4141-4151, 2013.

[2] J. Morris, R. Pramanik, X. Zhang et al., "Selenium- or quercetin-induced retardation of DNA synthesis in primary prostate cells occurs in the presence of a concomitant reduction in androgen-receptor activity," Cancer Letters, vol. 239, no. 1, pp. 111-122, 2006.

[3] Y. Ci, J. Qiao, and M. Han, "Molecular mechanisms and metabolomics of natural polyphenols interfering with breast cancer metastasis," Molecules, vol. 21, no. 12, p. 1634, 2016.

[4] K. W. Tan, Y. Li, J. W. Paxton, N. P. Birch, and A. Scheepens, "Identification of novel dietary phytochemicals inhibiting the efflux transporter breast cancer resistance protein (BCRP/ ABCG2)," Food Chemistry, vol. 138, no. 4, pp. 2267-2274, 2013.

[5] F. Farabegoli, A. Papi, G. Bartolini, R. Ostan, and M. Orlandi, "(-)-Epigallocatechin-3-gallate downregulates Pg-P and BCRP in a tamoxifen resistant MCF-7 cell line," Phytomedicine, vol. 17, no. 5, pp. 356-362, 2010.

[6] E. Langner and W. Rzeski, "Dietary derived compounds in cancer chemoprevention," Współczesna Onkologia, vol. 5, no. 5, pp. 394-400, 2012. 
[7] P. K. Goyal, R. Jain, S. Jain, and A. Sharma, "A Review on biological and phytochemical investigation of plant genus Callistimon," Asian Pacific Journal of Tropical Biomedicine, vol. 2, no. 3, pp. S1906-S1909, 2012.

[8] R. D. A. Raja, S. Jeeva, J. W. Prakash, J. M. Antonisamy, and V. Irudayaraj, "Antibacterial activity of selected ethnomedicinal plants from South India," Asian Pacific Journal of Tropical Medicine, vol. 4, no. 5, pp. 375-378, 2011.

[9] M. Z. Salem, H. M. Ali, N. A. El-Shanhorey, and A. AbdelMegeed, "Evaluation of extracts and essential oil from Callistemon viminalis leaves: antibacterial and antioxidant activities, total phenolic and flavonoid contents," Asian Pacific Journal of Tropical Medicine, vol. 6, no. 10, pp. 785-791, 2013.

[10] H. Ito, A. Saeed, and T. Yoshida, "Polyphenolic constituents of callistemon speciosus (natural medicine note)," Natural Medicines, vol. 54, no. 4, p. 204, 2000.

[11] M. I. Abdelhady and H. A. H. Aly, "Antioxidant antimicrobial activities of callistemon comboynensis essential oil," Free Radicals and Antioxidants, vol. 2, no. 1, pp. 37-41, 2012.

[12] I. Mahmoud, M. Marzouk, J. Moharram, J. Nolte, R. Fobbe, and M. Saleh, "Chemical composition of the Egyptian Callistemon lanceolatus DC. and Callistemon viminalis (gaertner loudan) oils," Bulletin of Faculty of Pharmacy, vol. 40, pp. 119-112, 2000.

[13] A. K. Jain, S. K. Dubey, M. S. Sikarwar, and S. K. Jain, "Hepatoprotective activity of methanolic extract of leaves of Callistemon lanceolatus," International Journal of Plant Sciences, vol. 2, no. 2, pp. 185-186, 2007.

[14] O. Oyedeji, O. Lawal, F. Shode, and A. Oyedeji, "Chemical composition and antibacterial activity of the essential oils of Callistemon citrinus and Callistemon viminalis from South Africa," Molecules, vol. 14, no. 6, pp. 1990-1998, 2009.

[15] R. A. Larayetan, O. O. Okoh, A. Sadimenko, and A. I. Okoh, "Terpene constituents of the aerial parts, phenolic content, antibacterial potential, free radical scavenging and antioxidant activity of Callistemon citrinus (Curtis) Skeels (Myrtaceae) from Eastern Cape Province of South Africa," BMC Complementary and Alternative Medicine, vol. 17, no. 1, p. 292, 2017.

[16] K. S. Soppimath, T. M. Aminabhavi, A. R. Kulkarni, and W. E. Rudzinski, "Biodegradable polymeric nanoparticles as drug delivery devices," Journal of Controlled Release, vol. 70, no. 1-2, pp. 1-20, 2001.

[17] I. Brigger, C. Dubernet, and P. Couvreur, "Nanoparticles in cancer therapy and diagnosis," Advanced Drug Delivery Reviews, vol. 64, pp. 24-36, 2012.

[18] S. Parveen and S. K. Sahoo, "Polymeric nanoparticles for cancer therapy," Journal of Drug Targeting, vol. 16, no. 2, pp. 108-123, 2008.

[19] Z. Li, H. Jiang, C. Xu, and L. Gu, "A review: using nanoparticles to enhance absorption and bioavailability of phenolic phytochemicals," Food Hydrocolloids, vol. 43, pp. 153-164, 2015.

[20] K. Kumbhani and Y. Agrawal, "Drug conjugated nanomedicine as prodrug carrier," Nanoscience \& NanotechnologyAsia, vol. 3, no. 1, pp. 86-94, 2013.

[21] S. Wang, R. Su, S. Nie et al., "Application of nanotechnology in improving bioavailability and bioactivity of diet-derived phytochemicals," Journal of Nutritional Biochemistry, vol. 25, no. 4, pp. 363-376, 2014.

[22] A. B. Shirode, D. J. Bharali, S. Nallanthighal, J. K. Coon, S. A. Mousa, and R. Reliene, "Nanoencapsulation of pomegranate bioactive compounds for breast cancer chemoprevention," International Journal of Nanomedicine, vol. 10, pp. 475-484, 2015.
[23] M. C. Pereira, D. A. Oliveira, L. E. Hill et al., "Effect of nanoencapsulation using PLGA on antioxidant and antimicrobial activities of guabiroba fruit phenolic extract," Food Chemistry, vol. 240, pp. 396-404, 2018.

[24] A. F. Ribeiro, C. T. G. Ferreira, J. F. dos Santos, L. M. Cabral, and V. P. de Sousa, "Design of experiments for the development of poly (d, l-lactide-co-glycolide) nanoparticles loaded with uncaria tomentosa," Journal of Nanoparticle Research, vol. 17, no. 2, p. 69, 2015.

[25] C. Gomes, R. G. Moreira, and E. Castell-Perez, "Poly (DLlactide-co-glycolide) (PLGA) nanoparticles with entrapped trans-cinnamaldehyde and eugenol for antimicrobial delivery applications," Journal of Food Science, vol. 76, no. 2, pp. N16-N24, 2011.

[26] B. N. Teixeira, N. Ozdemir, L. E. Hill, and C. L. Gomes, "Synthesis and characterization of nano-encapsulated black pepper oleoresin using hydroxypropyl beta-cyclodextrin for antioxidant and antimicrobial applications," Journal of Food Science, vol. 78, no. 12, pp. N1913-N1920, 2013.

[27] V. Vichai and K. Kirtikara, "Sulforhodamine B colorimetric assay for cytotoxicity screening," Nature Protocols, vol. 1, no. 3, pp. 1112-1116, 2006.

[28] M. R. El-Zahry, A. Mahmoud, I. H. Refaat, H. A. Mohamed, H. Bohlmann, and B. Lendl, "Antibacterial effect of various shapes of silver nanoparticles monitored by SERS," Talanta, vol. 138, pp. 183-189, 2015.

[29] P. Kolhar, A. C. Anselmo, V. Gupta et al., "Using shape effects to target antibody-coated nanoparticles to lung and brain endothelium," Proceedings of the National Academy of Sciences, vol. 110, no. 26, pp. 10753-10758, 2013.

[30] S. Barua, J.-W. Yoo, P. Kolhar, A. Wakankar, Y. R. Gokarn, and S. Mitragotri, "Particle shape enhances specificity of antibody-displaying nanoparticles," Proceedings of the $\mathrm{Na}$ tional Academy of Sciences, vol. 110, no. 9, pp. 3270-3275, 2013.

[31] L. Chen, S. Xiao, H. Zhu, L. Wang, and H. Liang, "Shapedependent internalization kinetics of nanoparticles by membranes," Soft Matter, vol. 12, no. 9, pp. 2632-2641, 2016.

[32] Y. Geng, P. Dalhaimer, S. Cai et al., "Shape effects of filaments versus spherical particles in flow and drug delivery," Nature Nanotechnology, vol. 2, no. 4, pp. 249-255, 2007.

[33] H. Wang, J. Feng, G. Liu, B. Chen, Y. Jiang, and Q. Xie, "In vitro and in vivo anti-tumor efficacy of 10-hydroxycamptothecin polymorphic nanoparticle dispersions: shape- and polymorphdependent cytotoxicity and delivery of 10-hydroxycamptothecin to cancer cells," Nanomedicine: Nanotechnology, Biology and Medicine, vol. 12, no. 4, pp. 881-891, 2016.

[34] B. Zhang, P. S. Lung, S. Zhao, Z. Chu, W. Chrzanowski, and Q. Li, "Shape dependent cytotoxicity of PLGA-PEG nanoparticles on human cells," Scientific Reports, vol. 7, no. 1, p. 7315, 2017.

[35] A. Platel, R. Carpentier, E. Becart, G. Mordacq, D. Betbeder, and F. Nesslany, "Influence of the surface charge of PLGA nanoparticles on theirin vitrogenotoxicity, cytotoxicity, ROS production and endocytosis," Journal of Applied Toxicology, vol. 36, no. 3, pp. 434-444, 2016.

[36] I. Nallamuthu, A. Parthasarathi, and F. Khanum, "Thymoquinone-loaded PLGA nanoparticles: antioxidant and anti-microbial properties," International Current Pharmaceutical Journal, vol. 2, no. 12, pp. 202-207, 2013.

[37] M. C. Pereira, R. S. Steffens, A. Jablonski et al., "Characterization and antioxidant potential of Brazilian fruits from the Myrtaceae family," Journal of Agricultural and Food Chemistry, vol. 60, no. 12, pp. 3061-3067, 2012. 
[38] M. C. Pereira, L. E. Hill, R. C. Zambiazi, S. Mertens-Talcott, S. Talcott, and C. L. Gomes, "Nanoencapsulation of hydrophobic phytochemicals using poly (dl-lactide-co-glycolide) (PLGA) for antioxidant and antimicrobial delivery applications: guabiroba fruit (Campomanesia xanthocarpa O. Berg) study," LWT-Food Science and Technology, vol. 63, no. 1, pp. 100-107, 2015.

[39] X. Xie, Q. Tao, Y. Zou et al., "PLGA nanoparticles improve the oral bioavailability of curcumin in rats: characterizations and mechanisms," Journal of Agricultural and Food Chemistry, vol. 59, no. 17, pp. 9280-9289, 2011.

[40] D. J. Dias, G. A. Joanitti, R. B. Azevedo, L. P. Silva, C. N. Lunardi, and A. J. Gomes, "Chlorambucil encapsulation into PLGA nanoparticles and cytotoxic effects in breast cancer cell," Journal of Biophysical Chemistry, vol. 6, no. 1, pp. 1-13, 2015.

[41] S. L. Gosangari and K. L. Watkin, "Effect of preparation techniques on the properties of curcumin liposomes: characterization of size, release and cytotoxicity on a squamous oral carcinoma cell line," Pharmaceutical Development and Technology, vol. 17, no. 1, pp. 103-109, 2012.

[42] M. M. Joseph, S. R. Aravind, S. K. George, R. K. Pillai, S. Mini, and T. T. Sreelekha, "Co-encapsulation of doxorubicin with galactoxyloglucan nanoparticles for intracellular tumortargeted delivery in murine ascites and solid tumors," Translational Oncology, vol. 7, no. 5, pp. 525-536, 2014.

[43] L. Ma, M. Kohli, and A. Smith, "Nanoparticles for combination drug therapy," ACS Nano, vol. 7, no. 11, pp. 9518-9525, 2013.

[44] S. Sampath, S. Subramani, S. Janardhanam, P. Subramani, A. Yuvaraj, and R. Chellan, "Bioactive compound 1,8-Cineole selectively induces $\mathrm{G} 2 / \mathrm{M}$ arrest in A431 cells through the upregulation of the p53 signaling pathway and molecular docking studies," Phytomedicine, vol. 46, pp. 57-68, 2018.

[45] R. Conte, A. Calarco, A. Napoletano et al., "Polyphenols nanoencapsulation for therapeutic applications," Journal of Biomolecular Research and Therapeutics, vol. 5, no. 2, 2016.

[46] Y. Davatgaran-Taghipour, S. Masoomzadeh, M. H. Farzaei et al., "Polyphenol nanoformulations for cancer therapy: experimental evidence and clinical perspective," International Journal of Nanomedicine, vol. 12, pp. 2689-2702, 2017.

[47] W. Li, J.-x. Wu, and Y.-y. Tu, "Synergistic effects of tea polyphenols and ascorbic acid on human lung adenocarcinoma SPC-A-1 cells," Journal of Zhejiang University SCIENCE B, vol. 11, no. 6, pp. 458-464, 2010.

[48] J. Kim, K.-M. Lee, E. Ko et al., "Berberine inhibits growth of the breast cancer cell lines MCF-7 and MDA-MB-231," Planta Medica, vol. 74, no. 1, pp. 39-42, 2008.

[49] Y. Liu, W. Cao, B. Zhang et al., "The natural compound magnolol inhibits invasion and exhibits potential in human breast cancer therapy," Scientific Reports, vol. 3, p. 3098, 2013.

[50] S. Javidfar, Y. Pilehvar-Soltanahmadi, R. Farajzadeh et al., "The inhibitory effects of nano-encapsulated metformin on growth and hTERT expression in breast cancer cells," Journal of Drug Delivery Science and Technology, vol. 43, pp. 19-26, 2018. 


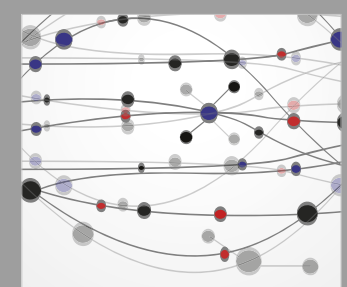

The Scientific World Journal
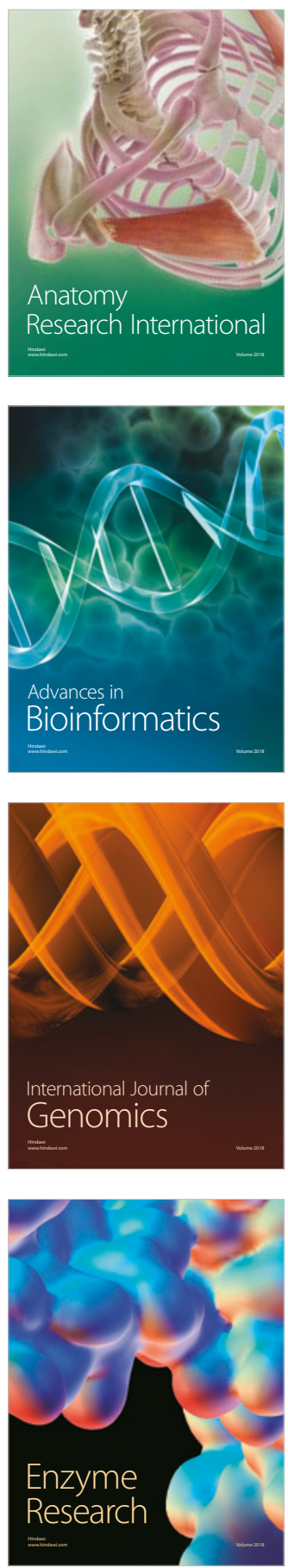
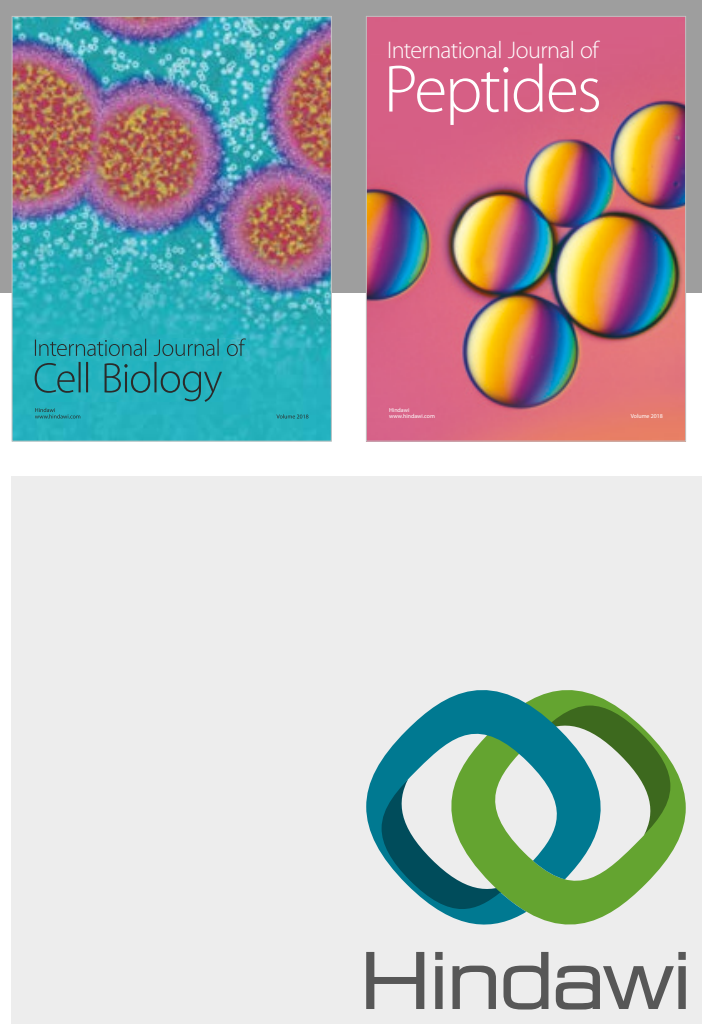

Submit your manuscripts at

www.hindawi.com
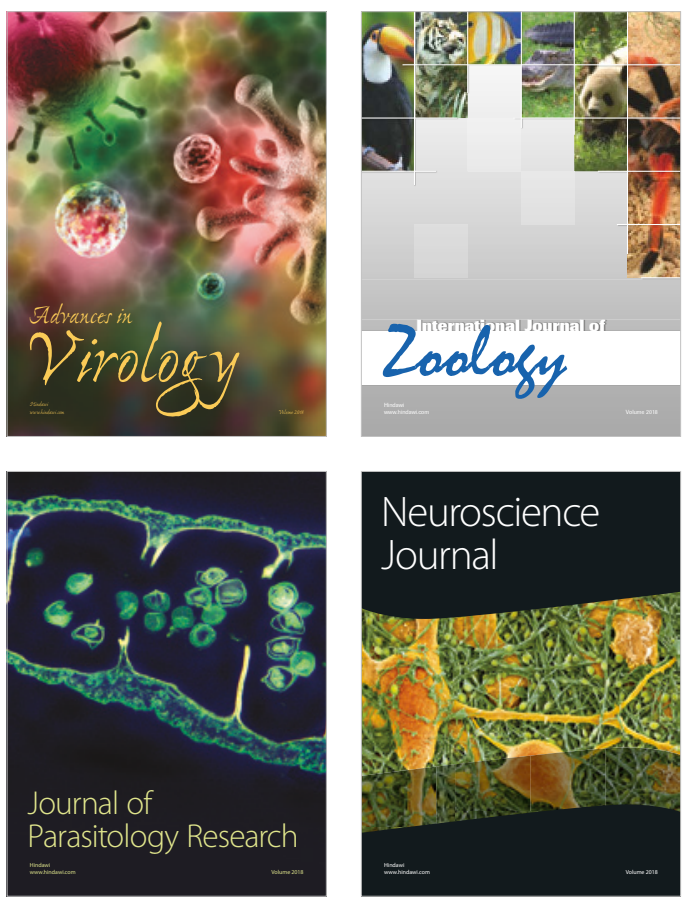
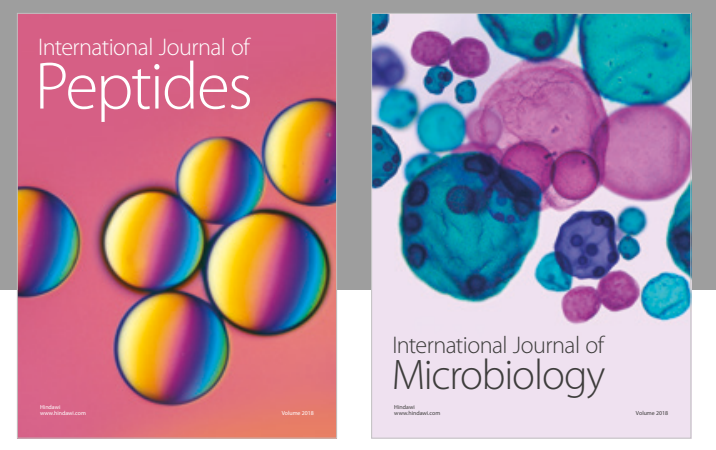

nternational Journal of Microbiology
Journal of
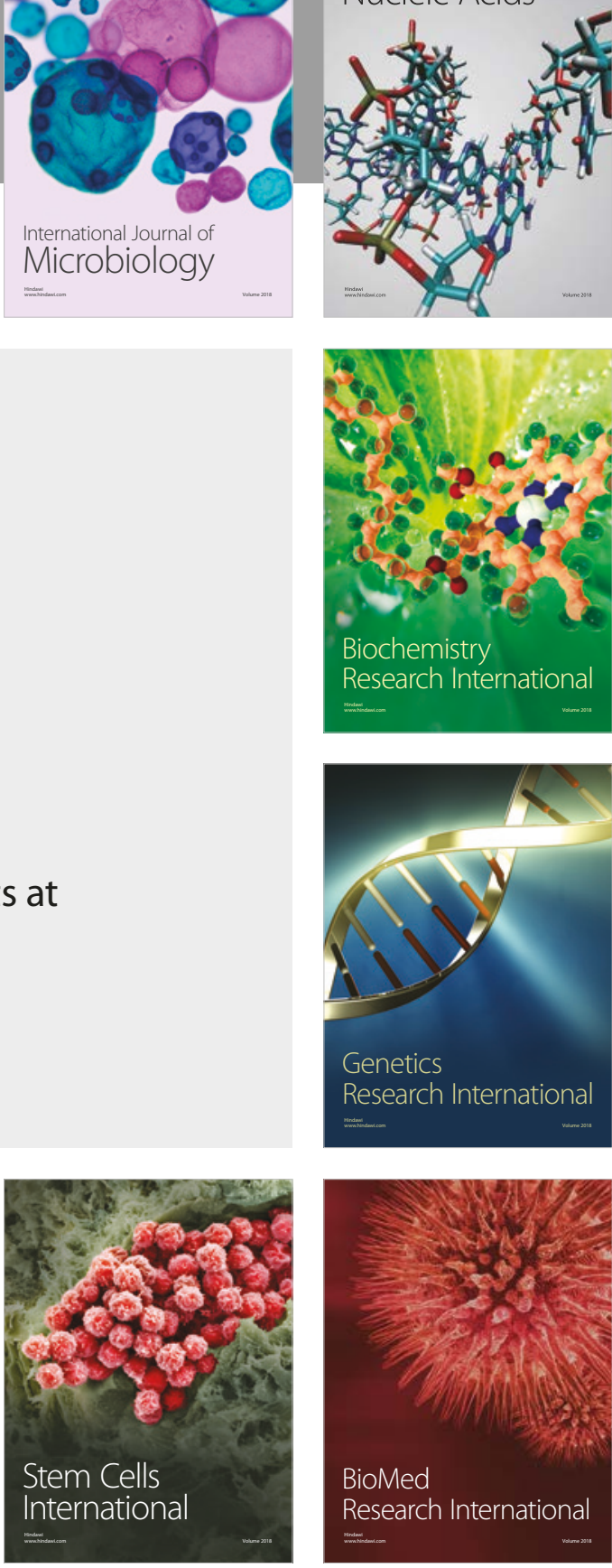
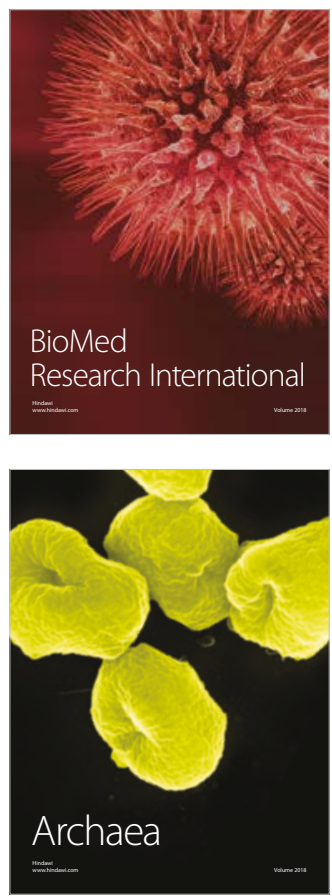